TM-1608

\title{
Improved Bunch Spreader Modules
}

\author{
Takao Ieiri* \\ Fermi National Accelerator Laboratory \\ P.O. Box 500, Batavia, Illinois
}

July 20, 1989 


\title{
IMPROVED BUNCH SPREADER MODULES
}

\author{
Takao Ieiri
}

July 20,1989

\section{Introduction}

In order to raise the threshold beam current of instabilities in the fixed target run, it is required to increase the longitudinal emittance, and hence the bunch length. The bunch spreader developed by G. Jackson [1] used a noise generator through a sharp-cut band pass filter (BPF) instead of a coherent signal. In that filter, the pass band frequency was near twice the synchrotron frequency $2 \mathrm{f}_{\mathrm{s}}$. The bandwidth of the filter was fixed between $350 \mathrm{~Hz}$ and $600 \mathrm{~Hz}$, though twice the synchrotron frequency changed from $500 \mathrm{~Hz}$ to $300 \mathrm{~Hz}$ after transition in Main Ring. The noise through the filter is applied to an RF amplitude modulator. In the Tevatron, where the synchrotron frequency $f_{s}$ varies from $120 \mathrm{~Hz}$ to $40 \mathrm{~Hz}$ during acceleration, the noise is applied to a phase shifter at the frequency of $f_{s}$. So, we need a tunable filter which tracks $2 f_{s}$ in the Main Ring and $f_{s}$ in the Tevatron.

This note describes details of improved bunch spreader modules using a tunable filter used in both the Main Ring and the Tevatron. A brief description about this module along with a beam test done in the Main Ring is shown in ref.[2].

\section{Tunable Filter}

Voltage tunable filters are commercially available, however, they are unsuitable for this project because their bandwidth is not flexible. Therefore, a modulation method which needs a mixer or a multiplier is employed to control the frequency band of the filter. Two signals with 
different frequencies are prepared, one is a noise signal coming from a noise generator through a fixed $\mathrm{BPF}$ and the other is a sine wave from a voltagecontrolled oscillator. They are applied to a multiplier and the output signal of the multiplier produces two frequency components at the sum and the difference frequencies of the input signals. Only the difference signal is used, the sum signal is filtered away.

\section{Circuit Description}

\subsection{General}

The block diagram of the module is shown in Figure 1. This module has a noise source tunable in frequency, amplitude and timing. The function and performance of each part will be described below. The prototype of the module has been built and is installed in a single width NIM module. Though the noise frequencies are different between the Main Ring and the Tevatron, the circuit boards are common for easy maintenance.

\subsection{Noise generator}

The noise source is the thermal noise of a resistor. Since the noise source voltage is very small, it is amplified with gain of about $80 \mathrm{~dB}$ by two operational amplifiers (TL084). The noise band is limited by the gainbandwidth of the amplifiers. The output of the noise generator is seen in Figure 2. The rms voltage of the noise is larger than an expected value from the thermal noise.

\subsection{High Q BPF}

In order to remove higher frequencies produced by the mixing, the pass band frequencies of the BPFs are around $2 \mathrm{kHz}$ in the Main Ring and around $560 \mathrm{~Hz}$ in the Tevatron. Since the pass band of the BPF is shifted to higher frequencies without changing a bandwidth, the BPF should have a higher $Q$ value. In order to realize such a filter using operational amplifiers (TL084), a 2nd order positive-feedback filter [3] is adopted. The advantage of this type of filter is the ability to change the center frequency and the $Q$ value independently. The details of this filter are explained in the Appendix. 
Three stages of 2 nd order filters makes 6 poles. The 1 st stage determines a lower cut-off frequency, the 2 nd the center frequency and the 3rd an upper cut-off one. Since the resonant frequency and the $Q$ value of each stage are controllable, the total bandwidth can be changed from 50 to $200 \mathrm{~Hz}$ in the Main Ring and from 10 to $30 \mathrm{~Hz}$ in the Tevatron within the ripple of $\pm 3 \mathrm{~dB}$. Typical spectra of the output of the high $Q B P F$ are seen in Figure 3. If a sharper filter is required, it is possible to change to four stages with 8 poles. The stability of the resonant frequency is acceptable.

\subsection{Voltage-Frequency Converter (VFC)}

The conversion coefficient of the VFC (Burr-Brown, VFC42) is changed to be $100 \mathrm{~Hz} / \mathrm{V}$ in the Main Ring and $20 \mathrm{~Hz} / \mathrm{V}$ in the Tevatron. The input voltage is expected to be 0 to $+10 \mathrm{~V}$. The output signal of the VFC is a square-wave of constant $25 \mu \mathrm{s}$ width, which contains many higher harmonics components. Stretching the pulse and a low pass filter reduce such components.

\subsection{1st Multiplier}

The two signals come into the 1st multiplier (AD534) as shown in Figure 1. This multiplier is used for frequency control. Several spectra are produced by the multiplier, however, the spectrum we need is a lowest one. A subsequent $\mathrm{BPF}$ reduces higher modes more than $20 \mathrm{~dB}$ below as seen in Figure 4.

\subsection{2nd and 3rd Multipliers}

The 2nd multiplier is used for amplitude control of the noise. It is necessary to give an external signal from 0 to $10 \mathrm{~V}$ to it. The 3 rd one is for gate control. The gate pulse is made from external two pulses, start and stop. The maximum gate time is set at the Timer to be $1.8 \mathrm{sec}$ in the Main Ring and 20sec in the Tevatron.

\subsection{APG Combiner}

The noise signal is summed with the RF voltage control (APG) and sent to the RF amplifier. In the Tevatron bunch spreader, however, the noise signal is sent to a phase shifter without the APG input. The noise signals 
without the APG input are shown in Figure 5. When the frequency control voltage $V_{f}$ changes, the noise band changes as shown in Figure 6-(a) and (b).

\section{Conclusion}

The bunch spreader works as a tunable noise source. The performance of the bunch spreader modules is summed up below.

Table 1. Performance of Modules

Main Ring Tevatron

$\begin{array}{lcc}\text { Frequency Range } & 0-900 \mathrm{~Hz} & 10-210 \mathrm{~Hz} \\ \text { Frequency Stability } & 0.8 \mathrm{~Hz} / \mathrm{C}^{\bullet} & 0.2 \mathrm{~Hz} / \mathrm{C}^{\bullet} \\ \text { F/V Conversion Rate } & 100 \mathrm{~Hz} / \mathrm{V} & 20 \mathrm{~Hz} / \mathrm{V} \\ \text { Bandwidth } & 50-200 \mathrm{~Hz} & 10-30 \mathrm{~Hz} \\ \text { Number of Poles } & 6 \text { or } 8 \text { (optional) } & 6 \text { or } 8 \text { (optional) }\end{array}$

\section{Acknowledgements}

The author would like to thank Dr. G. Jackson for bringing this project and giving helpful advice. He also thank members of Instrumentation Group for hospitality during his stay.

\section{References}

* Permanent address: KEK, Tsukuba-shi, Ibaraki-ken, 305, Japan

[1] G. Jackson, "Main Ring Bunch Spreaders: Past, 1987/1988 Fixed Target Run, and Proposed Future", TM-1572, February, 1989.

[2] G. Jackson and T. Ieiri, "Stimulated Longitudinal Emittance Growth in the Main Ring", to be published in Proceedings of the 1989 IEEE Particle Accelerator Conference and Fermilab-Conf-89/83.

[3] J.L. Hilburn and D.E. Johnson, "Manual of Active Filter Design", McGraw-Hill Co., (1973). 


\section{Appendix}

\section{Positive Feedback Band Pass Filter [3]}

A 2nd-order positive feedback type of $B P F$ is made up by two operational amplifiers as shown below. The first amplifier plays the multiple feedback type of $\mathrm{BPF}^{[3]}$ and the second one does positive feedback through the resistor $R_{3}$.

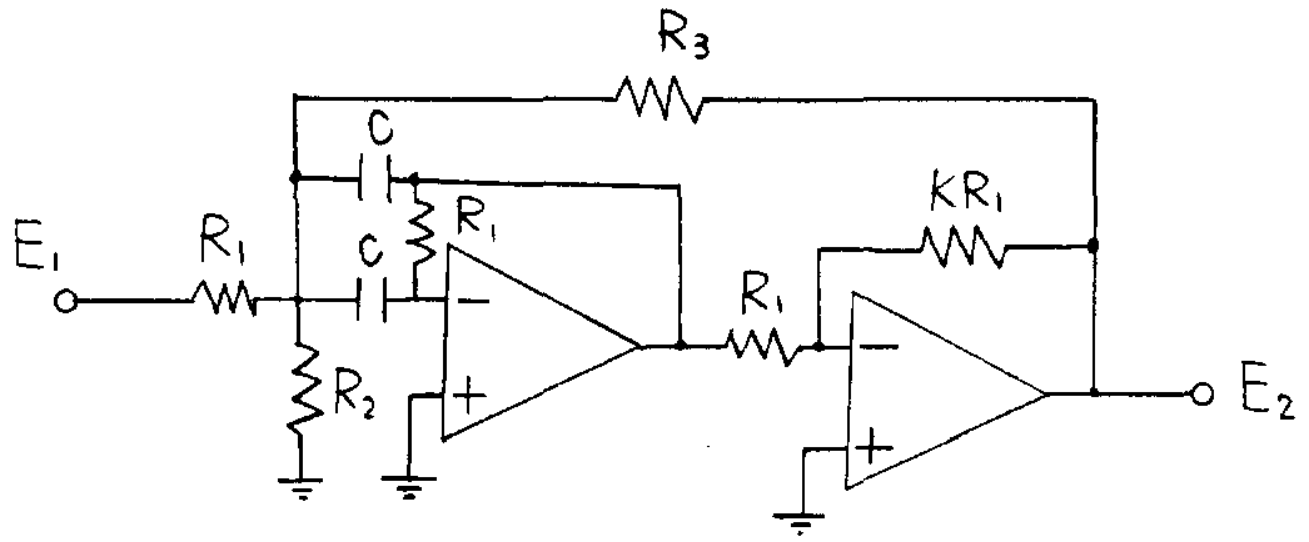

The voltage transfer function is given as

$$
\frac{\mathrm{E}_{2}}{\mathrm{E}_{1}}(\mathrm{~S})=\frac{\mathrm{S} \cdot \frac{\mathrm{K}}{\mathrm{R}_{1} \mathrm{C}}}{\mathrm{S}^{2}+\mathrm{S} \cdot \frac{1}{\mathrm{R}_{1}} \mathrm{C} \cdot\left[2-\frac{\mathrm{KR}}{\mathrm{R}_{3}} 1\right]+\frac{1}{\mathrm{C}^{2} \mathrm{R}_{1}} \cdot\left[\frac{1}{\mathrm{R}_{1}}+\frac{1}{\mathrm{R}_{2}}+\frac{1}{\mathrm{R}_{3}}\right]},
$$

where $s=j w$ and $w=2 \pi f$. The resonant frequency $f_{0}$, the bandwidth $\Delta f$ and the gain $G_{0}$ at $f_{0}$ are obtained from eq.(1).

$$
\begin{aligned}
f_{0} & =\frac{1}{2 \pi C} \cdot \sqrt{\frac{1}{R_{1}}\left[\frac{1}{R_{1}}+\frac{1}{R_{2}}+\frac{1}{R_{3}}\right]} \\
\Delta f & =\frac{1}{2 \pi R_{1} C} \cdot\left[2-\frac{K_{1}}{R_{3}}\right] \\
G_{0} & =\frac{K}{\left[2-\frac{K R_{1}}{R_{3}}\right]}
\end{aligned}
$$

Since $R_{2}$ is usually much smaller than $R_{1}$ and $R_{3}$, the $f_{0}$ can be changed by varying $R_{2}$ without changing $\Delta f$ and $G_{0}$. The quality factor or $\Delta f$ is adjusted by $K$ within the limit of $K R_{1}<2 R_{3}$. If $K R_{1} \geq 2 R_{3}$, the $B P F$ will oscillate. 


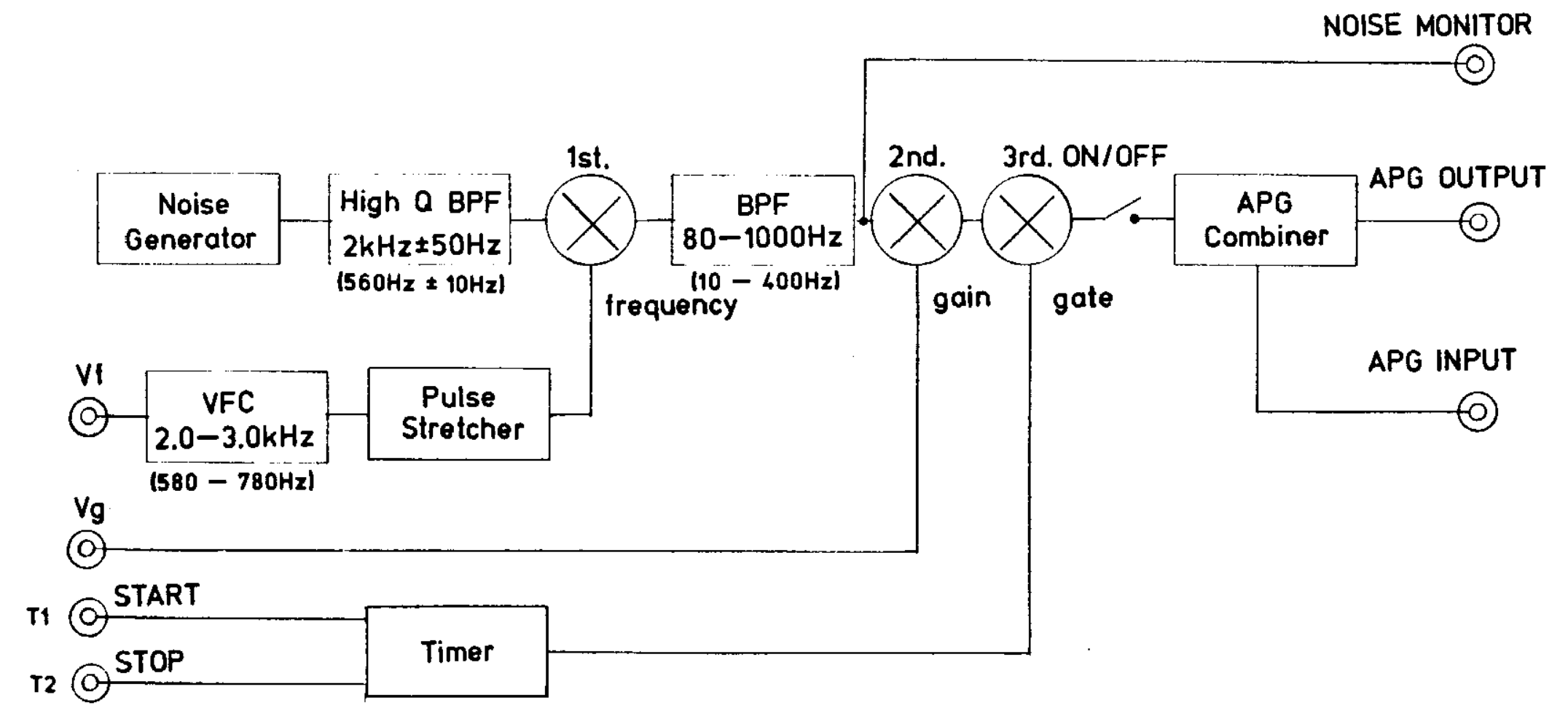

Figure 1. Block Diagram of Bunch Spreader, Values in parentheses are for Tevatron. 


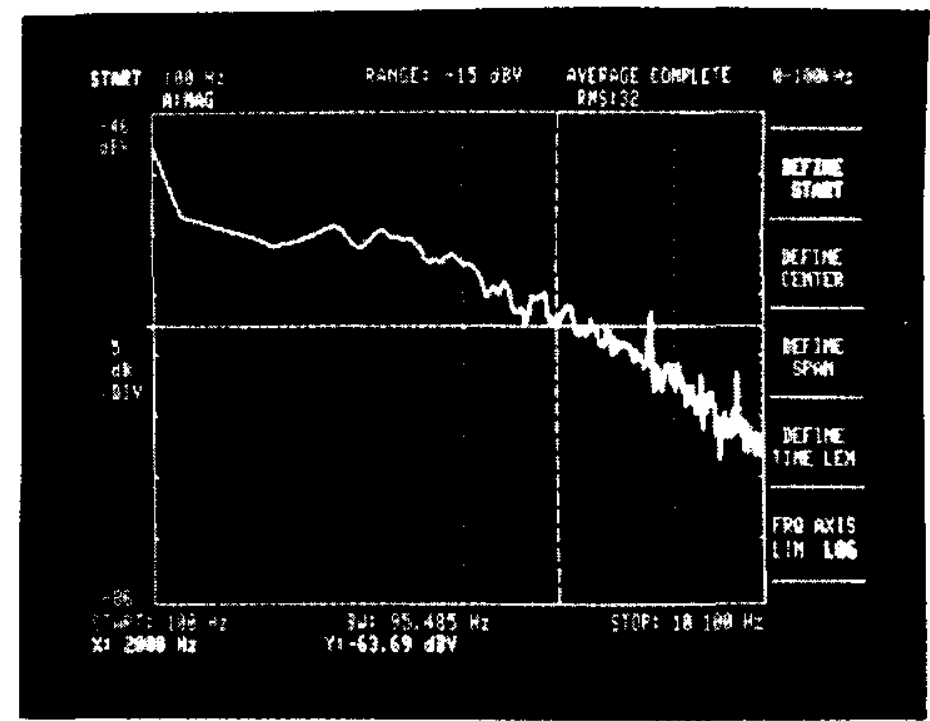

(a)

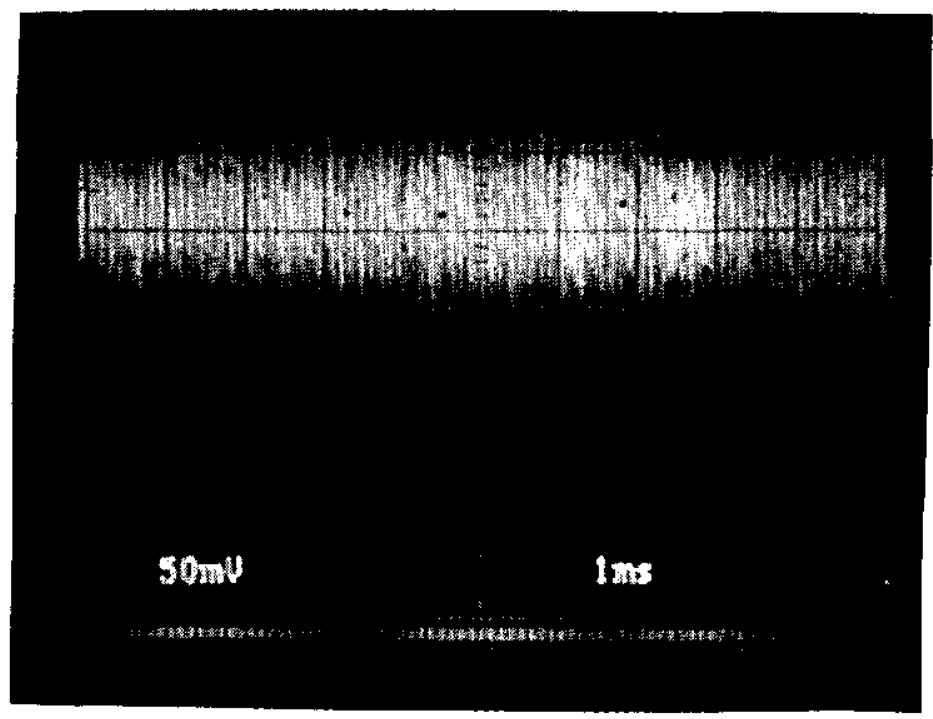

(b)

Figure 2. Output of Noise Generator, (a) in frequency domain, H: Log frequency from $100 \mathrm{~Hz}$ to $10 \mathrm{kHz}$, Bandwidth $=95 \mathrm{~Hz}, \mathrm{~V}: 5 \mathrm{~dB} / \mathrm{div}$, (b) in time domain, $\mathrm{H}: 1 \mathrm{msec} / \mathrm{div}, \mathrm{V}: 50 \mathrm{mV} /$ div. 


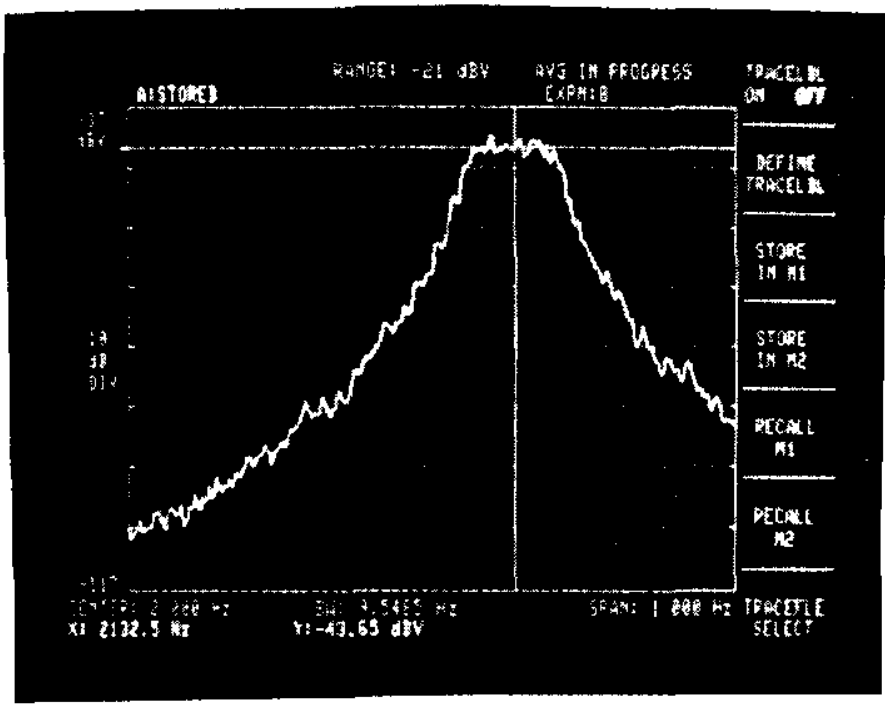

(a)

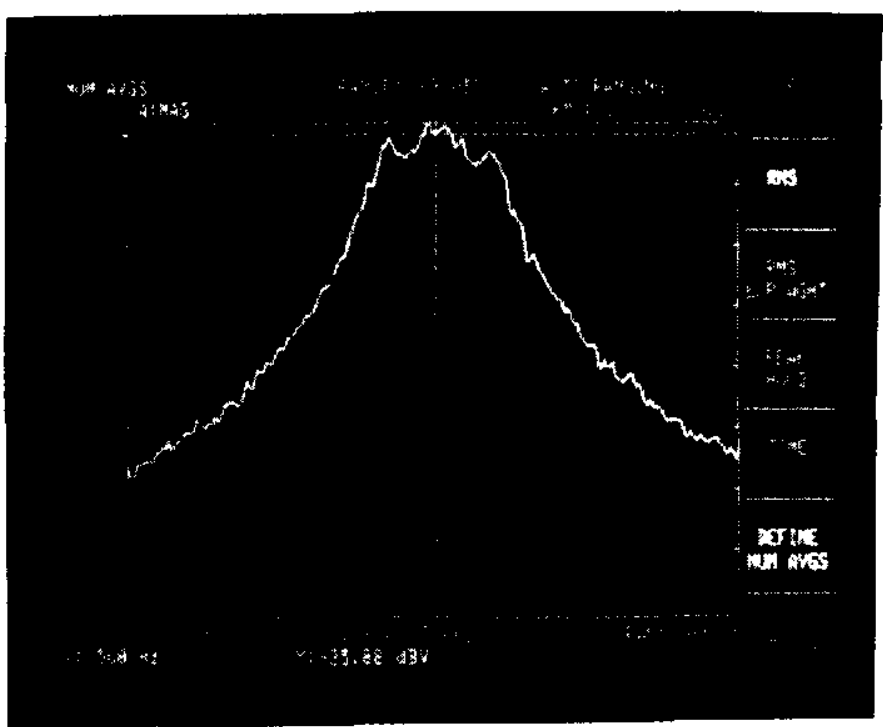

(b)

Figure 3. Output Spectra of High Q BPFs, (a) Main Ring, H: $100 \mathrm{~Hz} / \mathrm{div}, \mathrm{V}$ $10 \mathrm{~dB} / \mathrm{div}$, the center frequency is $2122.5 \mathrm{~Hz}$, (b) Tevatron, $\mathrm{H}: 10 \mathrm{~Hz} / \mathrm{div}, \mathrm{V}$ : $10 \mathrm{~dB} / \mathrm{div}$, the center frequency is $560 \mathrm{~Hz}$,. 


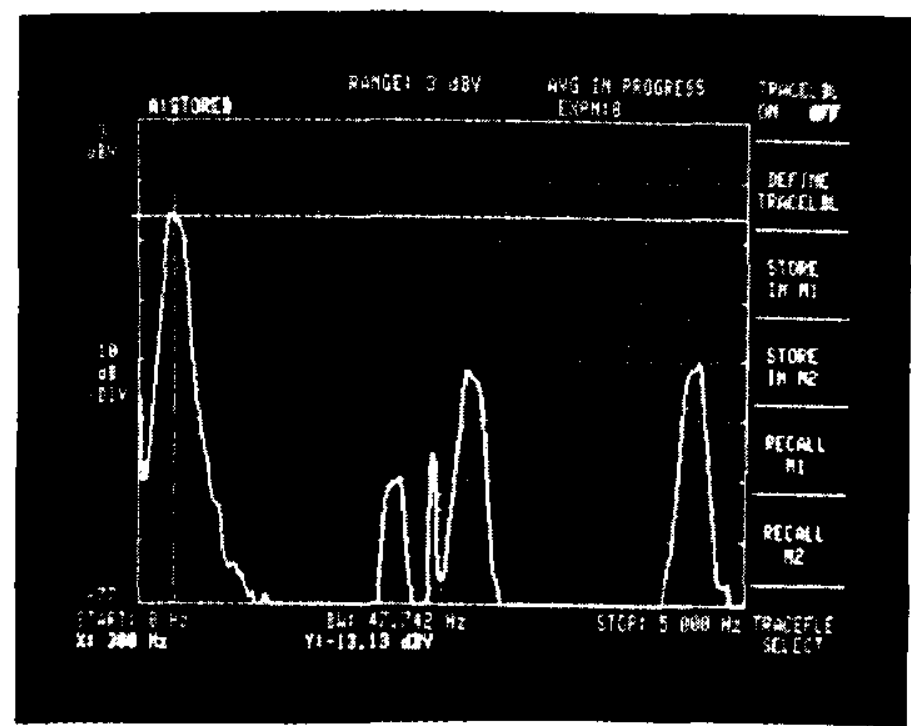

(a)

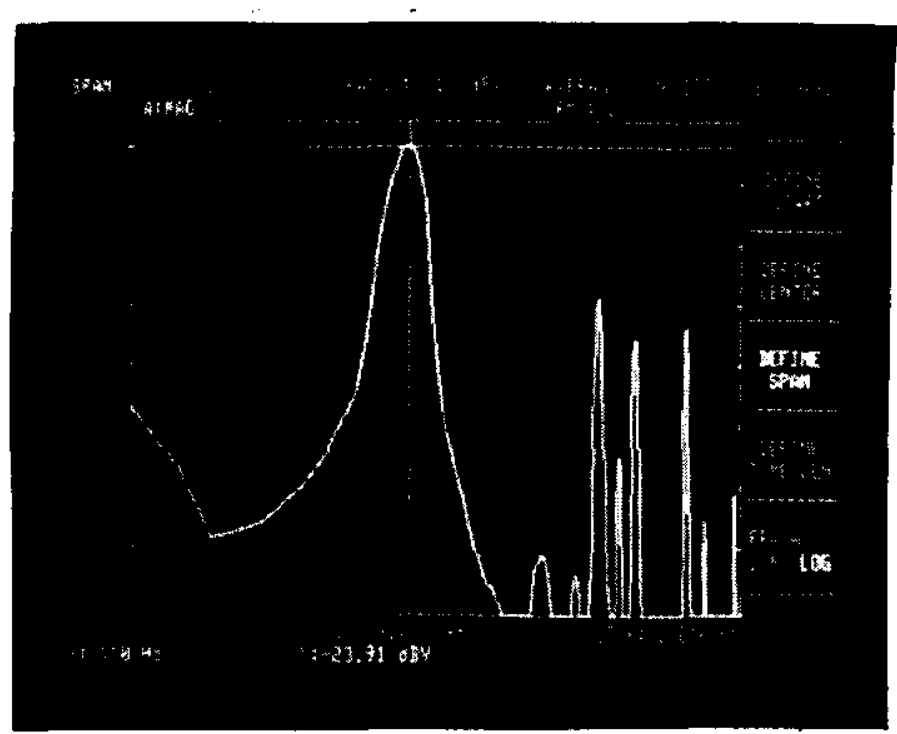

(b)

Figure 4. Outputs of BPF after the 1st Multiplier, (a) Main Ring at $\mathrm{V}_{\mathrm{f}}=7.0 \mathrm{~V}, \mathrm{H}: 500 \mathrm{~Hz} / \mathrm{div}, \mathrm{V}: 10 \mathrm{~dB} / \mathrm{div}$, (b) Tevatron at $\mathrm{V}_{\mathrm{f}}=5.0 \mathrm{~V}, \mathrm{H}: \log$ frequency from $10-2000 \mathrm{~Hz}, \mathrm{~V}: 10 \mathrm{~dB} /$ div. 


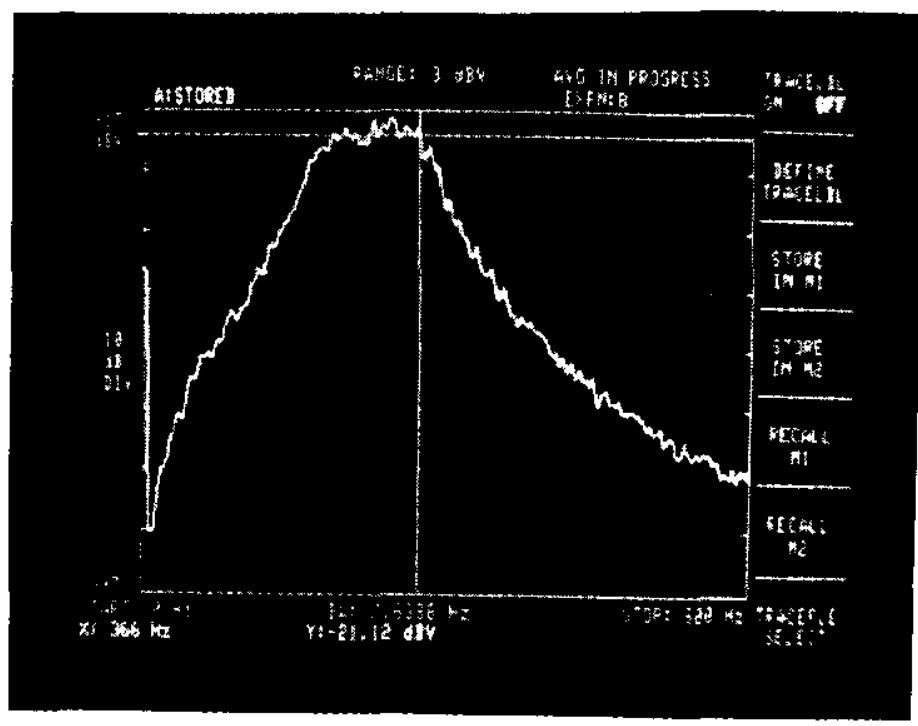

(a)

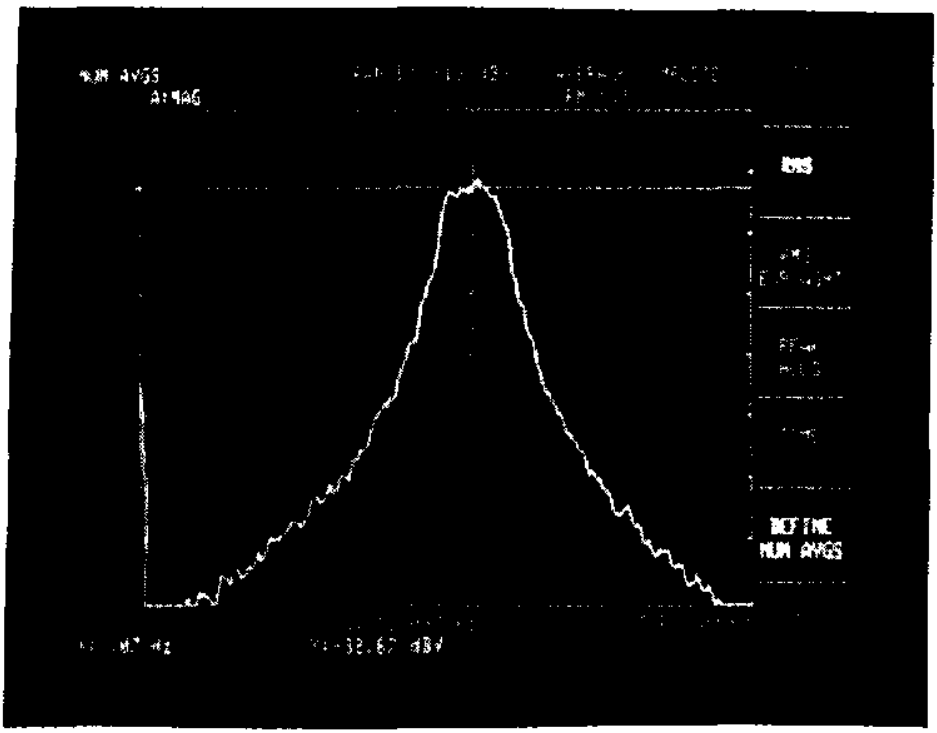

(b)

Figure 5. Typical Output Spectra, (a) Main Ring with band of $240-370 \mathrm{~Hz}$ when $\mathrm{V}_{\mathrm{f}}=7.0 \mathrm{~V}, \mathrm{H}: 100 \mathrm{~Hz} / \mathrm{div}, \mathrm{V}: 10 \mathrm{~dB} / \mathrm{div}$, (b) Tevatron with band of 98 $115 \mathrm{~Hz}$ when $\mathrm{V}_{\mathrm{f}}=5.0 \mathrm{~V}, \mathrm{H}: 20 \mathrm{~Hz} / \mathrm{div}, \mathrm{V}: 10 \mathrm{~dB} / \mathrm{div}$. 


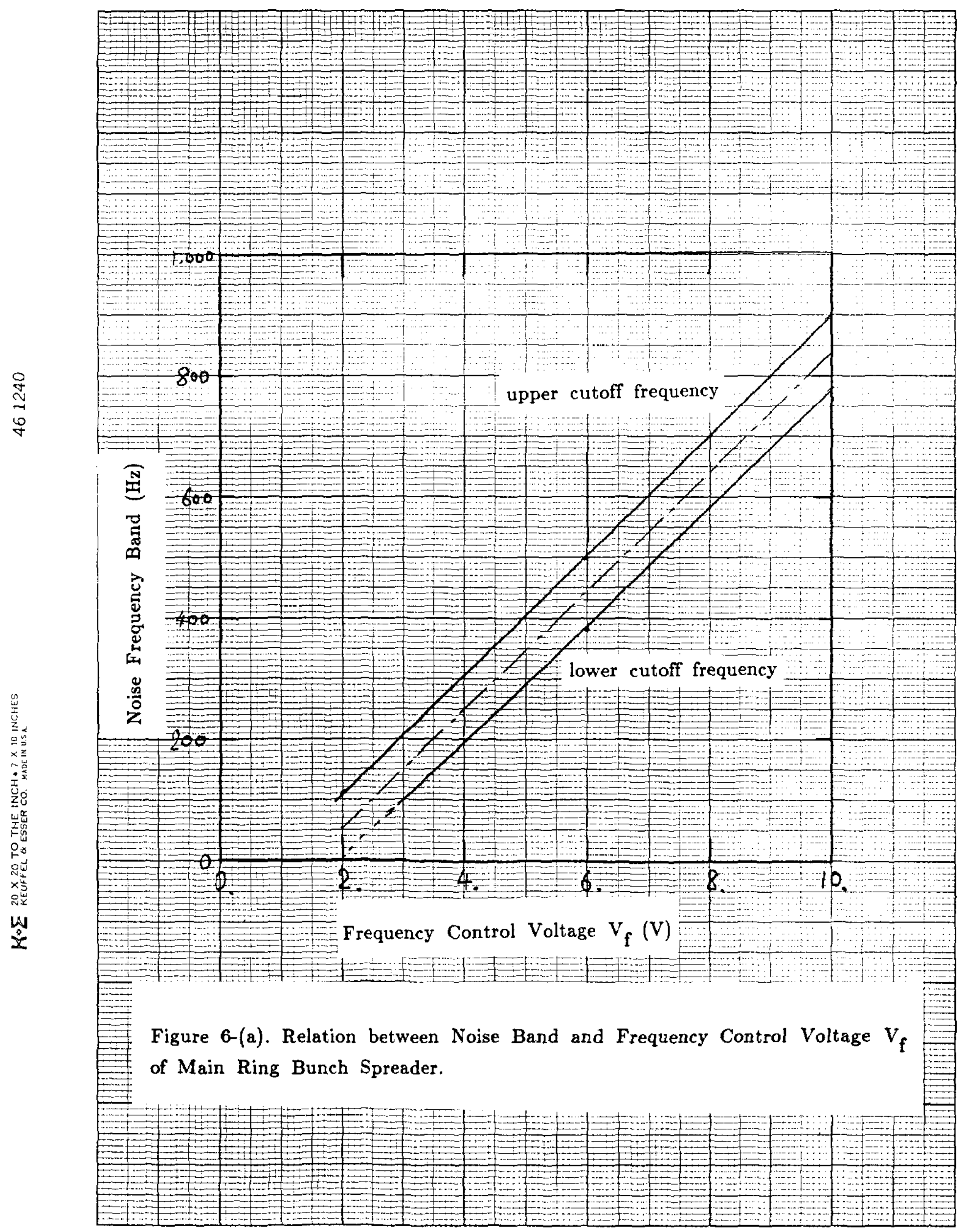

$$
-11-
$$




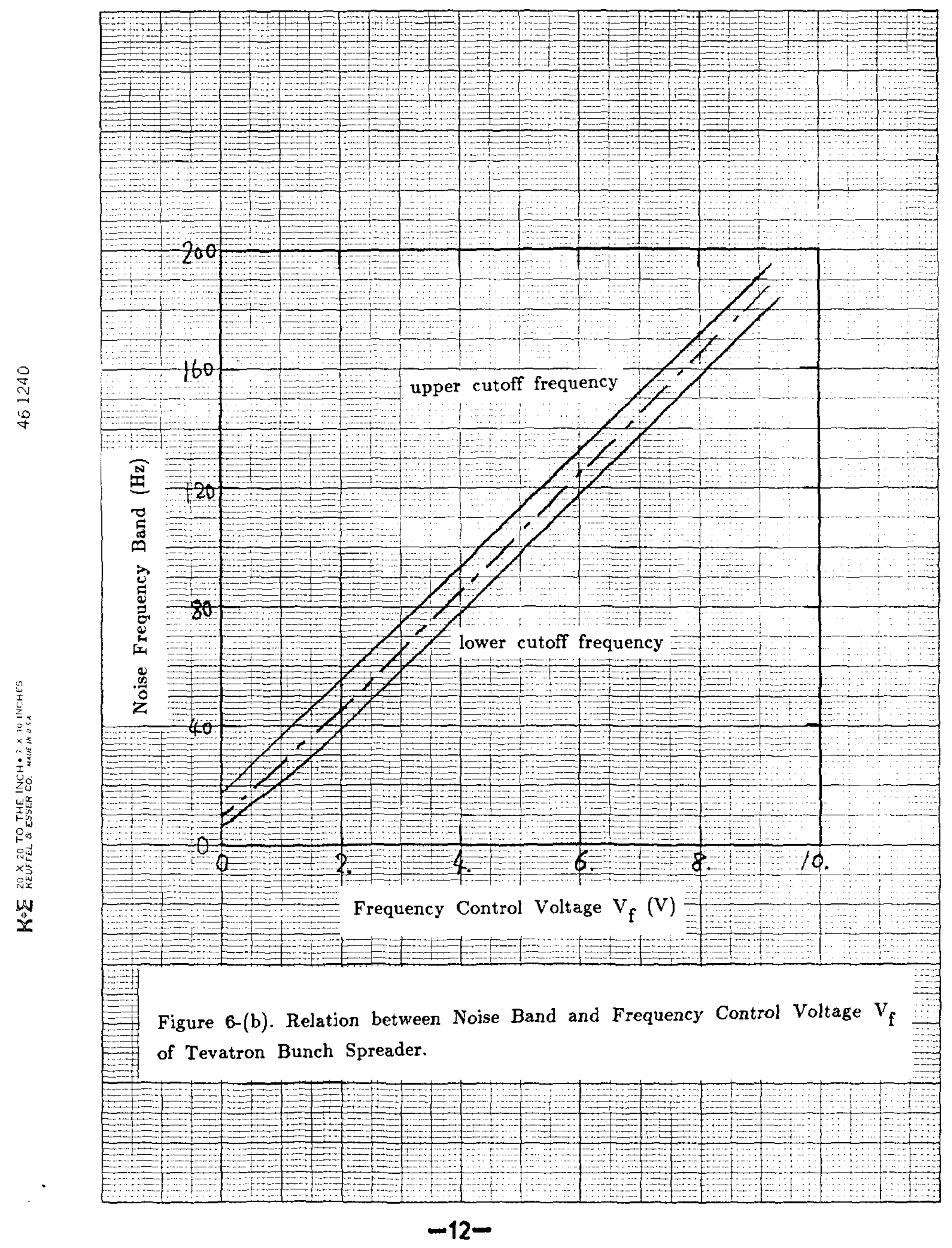

